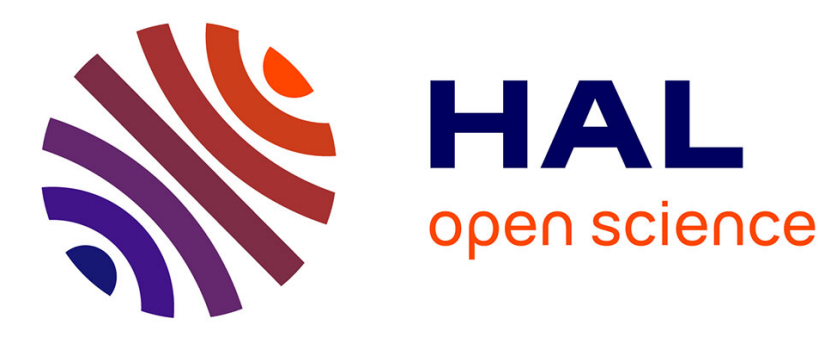

\title{
Pyrrolizidine alkaloids in food: Downstream contamination in the food chain caused by honey and pollen
}

Till Beuerle, Michael Kempf, Maximilian Wittig, Kirsten Schönfeld, Luise Cramer, P Schreier

\section{To cite this version:}

Till Beuerle, Michael Kempf, Maximilian Wittig, Kirsten Schönfeld, Luise Cramer, et al.. Pyrrolizidine alkaloids in food: Downstream contamination in the food chain caused by honey and pollen. Food Additives and Contaminants, 2010, pp.1. 10.1080/19440049.2010.521771 . hal-00633998

\section{HAL Id: hal-00633998 https://hal.science/hal-00633998}

Submitted on 20 Oct 2011

HAL is a multi-disciplinary open access archive for the deposit and dissemination of scientific research documents, whether they are published or not. The documents may come from teaching and research institutions in France or abroad, or from public or private research centers.
L'archive ouverte pluridisciplinaire HAL, est destinée au dépôt et à la diffusion de documents scientifiques de niveau recherche, publiés ou non, émanant des établissements d'enseignement et de recherche français ou étrangers, des laboratoires publics ou privés. 


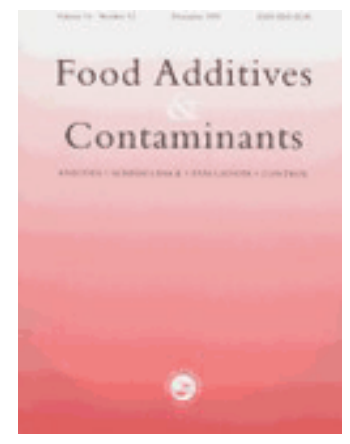

\section{Pyrrolizidine alkaloids in food: Downstream contamination in the food chain caused by honey and pollen}

\begin{tabular}{|r|l|}
\hline Journal: & Food Additives and Contaminants \\
\hline Manuscript ID: & TFAC-2010-131.R2 \\
\hline Manuscript Type: & Original Research Paper \\
\hline Author: & 30 -Aug-2010 \\
\hline & $\begin{array}{l}\text { Complete List of Authors: } \\
\text { Kemphö, Michael } \\
\text { Sittig, Maximilian } \\
\text { Cramer, Luise; TU Braunschweig, Institut f. Pharmazeutische } \\
\text { Biologie } \\
\text { Schreier, P }\end{array}$ \\
\hline Methods/Techniques: & Chromatography - GC/MS, Clean-up \\
\hline Additives/Contaminants: & Natural toxicants, Natural toxicants - alkaloids \\
\hline Food Types: & Honey, Plants, Processed foods \\
\hline & \\
\hline
\end{tabular}

\section{SCHOLARONE ${ }^{\text {M }}$ Manuscripts}


Keywords: Food safety, pyrrolizidine alkaloids, pyrrolizidine $\mathrm{N}$-oxides, gas chromatography mass spectrometry (GC-MS), honey, pollen, carry-over.

\begin{abstract}
Abbreviations:
$\mathrm{EH}$, experimental honey; EIMS SIM mode, electron ionisation mass spectrometry single ion monitoring mode; HRGC-MS, high resolution gas chromatography-mass spectrometry; LLE, continuous liquid-liquid extraction; LOQ, limit of quantification; MSTFA, $N$-Methyl- $N$ (trimethylsilyl)trifluoroacetamide; PA, pyrrolizidine alkaloid; PANOs, pyrrolizidine alkaloid $\mathrm{N}$ oxides; SD, standard deviation; S/N, signal-to-noise ratio; SPE, solid phase extraction; SCX, strong cation exchange; SIM, single ion monitoring; TMS, trimethylsilyl group.
\end{abstract}




\section{Introduction}

Recently, the European Food Safety Agency (EFSA) published an opinion on pyrrolizidine alkaloids (PAs) in animal feed (EFSA 2007). All major aspects and recent developments of PA-occurrence and toxicity to humans and livestock are covered. In addition, carry-over of PAs through livestock into food like milk, eggs and honey are mentioned. EFSA concludes, that "the contribution of the residues (here: PAs) in animal derived tissues to human exposure is low; however, honey, in which PA residues are regularly found, deserves special attention" (EFSA 2007).

Very recently, several independent studies, applying different analytical methods have demonstrated the frequent occurrence of PAs in honey and pollen (for review see Kempf et al. 2010a). Compared to other food types, like milk, flour, eggs or meat, the data pool of PA-occurrence in honey is growing steadily since German honey packers are addressing the problem and many batches of raw honey are monitored for PA-occurrence during the import process (Lüllmann 2010).

Besides herbal products made of PA-plants or being contaminated with PA-plants, it seems evident, that honey (and pollen, although not really accessible, since there is neither a clear product definition nor solid information on consumption or production quantities) shows a relatively high degree of PA-contamination. This is because these products are still very close to the original plant source only diluted to some extend during honey collection or packing. Other food types, as mentioned by EFSA are expected to show lower PA-concentrations (EFSA 2007). Addressing PA-contamination of food as a basis for an appropriate risk assessment might therefore require several strategies. For one it seems necessary to increase the data basis for many different types of food like milk, eggs, flour etc. In addition, it would be wise to check whether PAs from PA-contaminated food such as honey/pollen may represent a risk for further downstream contamination in the food chain. Interestingly, honey in food recipes is associated by consumers or 
producers with attributes of quality, health, "natural" or especially appropriate for kids nutrition. In cases where PAs can survive technological food processing this might increase the variety or change the order of foods that should be analyzed for a preventive risk assessment.

Hence, we have adapted our existing methods to investigate the persistence or disappearance of PAs in processed foods that contain honey as an ingredient.

In addition we addressed a question that is under active discussion in the published literature on PA-contamination of honey and pollen, i.e. whether pollen from PA-plants is the only source of PA-contamination of honey (Boppré et al. 2005; Boppré et al. 2008; Kempf et al. 2010a; Kempf et al. 2010b). This scenario would, at least to some degree, offer the possibility that legally permitted filtration of PA-pollen containing honey could result in a significant lower PA-content of the filtered honey. 


\section{Material and Methods}

\section{Chemicals and Solvents}

All chemicals were of analytical reagent purity and purchased from Fluka (Buchs, Switzerland), Merck (Darmstadt, Germany), Roth (Karlsruhe, Germany), Acros Organics (Geel, Belgium) and Sigma-Aldrich (Steinheim, Germany). Solvents were of HPLC grade purity, other solvents were redistilled before use.

\section{Reference materials}

To evaluate the analytical method six PAs, monocrotaline (Sigma-Aldrich, Steinheim, Germany), senecionine (Roth, Karlsruhe, Germany), seneciphylline (Roth, Karlsruhe, Germany), senkirkine (Roth, Karlsruhe, Germany), heliotrine (Latoxan, Valence, France) and retrorsine (Sigma-Aldrich, Steinheim, Germany) were purchased.

\section{PA determination of honey-containing foods}

\section{Sample material}

Honey-containing foods were purchased from supermarkets in Germany and from German internet shops. The sample list comprised 60 individual samples including mead $(n=20)$, candy $(n=10)$, fennel honey $(n=9)$, soft drinks/juices $(n=9)$, power bars and cereals $(n=7)$, jelly babies $(n=3)$, baby food $(n=3)$ supplements $(n=3)$ and fruit sauce $(n=1)$. All samples showed a minimum honey content of $5 \%$.

\section{Sample preparation}

General

Since heliotrine, the internal standard used in this study, is a natural occurring PA, each analysis had to be performed a priori in duplicates (cf. Kempf et al. 2008). Due to the different textures and differing honey-content of the foods, some method parameters of the general work-up had to be modified accordingly. 


\begin{abstract}
Mead and fennel honey
Duplicates (approx. $45 \mathrm{~g}$, weighed to the nearest $0.01 \mathrm{~g}$ ) were analyzed with the published method for PA-analysis in honey (cf. Kempf et al. 2008)
\end{abstract}

\title{
Other foodstuff
}

The amount of each individual sample used for the work-up had to be calculated according to the honey content mentioned in the ingredient list to achieve $20 \mathrm{~g}$ of honey per work-up. Liquid foods were diluted with water to $1000 \mathrm{ml}$ and acidified with $0.5 \mathrm{M} \mathrm{H}_{2} \mathrm{SO}_{4}$. Solid foods, e.g. candies, were dissolved in water, acidified with $0.5 \mathrm{M} \mathrm{H}_{2} \mathrm{SO}_{4}$ and diluted to a total volume of $1000 \mathrm{ml}$. After addition of $2 \mathrm{~g}$ zinc dust to each solution the mixture was stirred at room temperature for $3 \mathrm{hrs}$. Samples were centrifuged $(2250 \times \mathrm{g})$ for $6 \mathrm{~min}$ and the supernatants filtered afterwards. The filtrates were alkalized with $\mathrm{NaOH}(10 \%, w / w)$ to $\mathrm{pH}=11$. The alkaline solutions were subjected to continuous liquid-liquid extraction (LLE) for $48 \mathrm{hrs}$ with $210 \mathrm{ml}$ of a pentane-dichloromethane mixture $(2: 1 ; \mathrm{v} / \mathrm{v})$. The organic extract was dried over anhydrous sodium sulphate, filtered, and carefully concentrated to approximately $5 \mathrm{ml}$ on a Vigreux column $\left(40^{\circ} \mathrm{C}\right)$. The organic residue was dried under a gentle flow of nitrogen and resolved in an ultrasonic bath with $50 \mathrm{ml}$ of $0.05 \mathrm{M} \mathrm{H}_{2} \mathrm{SO}_{4}$. This solution was applied to preconditioned HF Bond Elut LRC (500 mg) strong cation exchange columns (Varian, Palo Alto, CA, USA) and the sample was further processed as previously described (cf. Kempf et al. 2008).

\section{Honey filtration experiments}

\section{Sample material}

A PA-free rape honey was slowly warmed to $35^{\circ} \mathrm{C}$ on a water bath. Floral Senecio vernalis pollen (pollen PA-content: $1.85 \pm 0.13 \mathrm{mg} \mathrm{g}^{-1}$, calculated as retronecine equivalents; $c f$. Kempf et al. 2010b) was added to $1000 \mathrm{~g}$ honey and was stirred until a homogenous 
distribution was ensured. Three experimental honeys $(\mathrm{EH})$ with different dosages of PA pollen $\left(\mathrm{EH} 1=3 \mathrm{mg} \mathrm{kg}^{-1} ; \mathrm{EH} 2=30 \mathrm{mg} \mathrm{kg}^{-1}\right.$ and $\left.\mathrm{EH} 3=300 \mathrm{mg} \mathrm{kg}^{-1}\right)$ were obtained. The resulting EHs were stored at room temperature in the dark. $20 \mathrm{~g}$ of honey (triplicates each) were sampled at defined time points: day 1,6 wks and 12 wks (day 1, only as unfiltered sample) and were analyzed for PA-content after filtration. A second set of honey samples was analyzed without filtration.

\section{Filtration and sample preparation}

The apparatus consisted of a brazen mantle that allowed a circulating water heating. Centred in this mantle a $20 \mathrm{ml}$ syringe with Luer-lock-fitting was seated in an accurately fitting hole of the mantle. Underneath the brazen mantle a $5 \mu \mathrm{m}$ cellulose-nitrate syringefilter (Puradisc ${ }^{\mathrm{TM}}$ FP30/5.0 CN, Whatman, Dassel, Germany) was attached to the syringe. The experimental filtration parameters were adapted from industrial parameters for honey filtration (Paine et al. 1934; Crane 1979; Beckmann 2008). The filtration apparatus was preconditioned with a water bath circulation for $10 \mathrm{~min}$ at $80^{\circ} \mathrm{C} .20 \mathrm{~g}$ of each experimental honey (EH1 to $\mathrm{EH} 3$ ) was diluted with $30 \mathrm{ml} 0.05 \mathrm{M} \mathrm{H}_{2} \mathrm{SO}_{4}, 2 \mu \mathrm{g}$ heliotrine was added as internal standard (50 $\mu \mathrm{L}$ of a heliotrine standard solution with $40 \mathrm{ng}^{-1} \mathrm{~L}^{-1}$ methanol) then applied to the filtration apparatus. Filtration was started after an equilibration time of 2 min by slightly pushing down the plunger. The overall procedure was finished in less then 10 min and the collected filtrate was processed like regular honey (cf. Kempf et al. 2008). Unfiltered samples were analyzed like regular honey (cf. Kempf et al. 2008).

\section{HRGC-MS}

GC-MS was carried out with a Fisons Instruments GC 8060 (Thermo Electron, Dreieich, Germany) gas chromatograph with split/splitless injection $\left(220^{\circ} \mathrm{C} / 1: 20\right)$ directly coupled to a Fisons Instruments MD 800 mass spectrometer (Thermo Electron, Dreieich, Germany) essentially as described in Kempf et al. 2008. 


\section{Quantification}

Standard controlled relative quantification with heliotridine (originated from $2 \mu \mathrm{g}$ heliotrine per sample) as internal standard was performed by HRGC-MS under the above mentioned conditions. Linear retention indices were 1600 and 1632 for di-TMS-retronecine and the standard di-TMS-heliotridine, respectively. Integration of peak area counts in EIMS SIM mode $(\mathrm{m} / \mathrm{z} 93,183$ and 299$)$ was carried out. The relative intensities of these analytical ions to each other were used as tool for the determination of the purity of the corresponding peak. These values were compared to values obtained from authentic reference compounds; variances of $<10 \%$ were tolerated. No extraction/response factors $(F=1.0)$ were considered. The data obtained was finally calculated into retronecine equivalents as previously described in Kempf et al. 2008. 


\section{Results and Discussion}

\section{Sample preparation, recovery rate and $L O Q$}

All samples were retail goods obtained from supermarkets in Germany or via the internet from German internet shops. The sample list comprised 60 individual samples including mead $(n=20)$, candy $(n=10)$, fennel honey $(n=9)$, soft drinks $(n=9)$, power bars and cereals $(n=7)$, jelly babies $(n=3)$, baby food $(n=3)$, supplements $(n=3)$ and fruit sauce $(n=1)$. The samples were selected based on a minimum honey content of $>5 \%$ according to the ingredients list or according to production guidelines for these products.

Fennel honey is not honey in the sense of the German food regulation. This food is not clearly defined but may contain substantial amounts of honey (up to $37 \%$ ) but also different other sugar types or syrup. Honey is also a major ingredient of mead, which is defined in German guidelines for wine and sparkling wine related drinks and spirits to contain 1 part honey and 2 parts water (GMBI 2003) and is produced by full or partial fermentation. These two foods were analyzed usually straight forward with our routine method for PA analysis in honey (Kempf et al. 2008).

Solid foods and foodstuff containing lower amounts of honey required a different work-up strategy. As a general approach the sample was dissolved/diluted or clarified, the possible PANOs were reduced by a zinc dust treatment and the resulting solutions were exhaustively extracted (48 hrs) by dichloromethane : pentane (1:2; v/v) in a LLE-step. This was necessary to prevent a matrix overloading of the SCX-SPE cartridges that were used in the subsequent solid phase concentration. The combination of the LLE preconcentration and SCX-SPE permitted the analysis of higher sample weights/volumes of food products which was necessary to increase the weight of food to get the amount that represented $20 \mathrm{~g}$ of honey.

During the establishment of the procedure it came into notice, that a thorough LLE is the key step in this protocol. The newly introduced LLE-step in the work-up procedure 
compared to regular honey work-up was checked with a set of standard PAs dissolved in honey matrix to check recovery rates and reproducibility. These results are summarized in Figure 1.If the extraction time was $24 \mathrm{hrs}$, the recovery rate was significantly lower (56 to $70 \%)$ and the deviation in reproducibility rate much higher (21-34\%) as compared to 78 $88 \%$ (recovery rate) and $5-11 \%$ (deviation in reproducibility) for 48 hrs extractions. By increasing the amount of initial sample weight and additional expenditure during sample clean-up the $L O Q$ could be maintained at $0.01 \mathrm{ppm}(\mathrm{S} / \mathrm{N}=7: 1)$ as reported earlier (Kempf et al. 2008; Kempf et al. 2010b) and the varied method could be applied successfully to all different kinds of matrices.

\section{PA-content of honey containing food}

For the determination of the PA-concentrations of the analyzed samples, recovery rates were not considered since the internal standard heliotrine was added before the work-up procedure and it was assumed that due to structural similarity equal losses for the standard and the analytes occurred during work-up and derivatization. In addition, the analyte (di-TMS-retronecine) and the internal standard derivative (di-TMS-heliotridine) are diastereomeric and have identical mass spectra. Hence, a response factor for these signals was not considered (see also Kempf et al. 2008). As a consequence of the use of heliotrine (a natural occurring heliotrinde-PA) all samples were processed in duplicates (cf. Kempf et al. 2008), one with internal standard and one without. In concrete terms, even if the sample without internal standard was tested negative for retronecine-type PAs the internal standard had to be detected in the other sample. Hence the latter one served always as a control for the reliability and sensitivity of the overall method.

In our study, eight out of 60 honey-containing food samples were tested PA-positive. The results are summarized in Figure 2. Positive samples were found in three food classes, mead, candy and fennel honey. 
Since fennel honey is basically regular honey with added ingredients, the PA-positive samples there can be easily explained and were somewhat expected.

Even so, honey is only an ingredient in the range of 5 to $37 \%$ in all the investigated products, $13 \%$ of the tested products were PA-positive and the average PA-content was $0.10 \mu \mathrm{g} \mathrm{g}^{-1}$. Our recent study on retail honey showed 9\% PA-positive samples and an average of $0.06 \mu \mathrm{g} \mathrm{g}^{-1}$ hence slightly lower values (Kempf et al. 2008).

Additionally, the PA-concentration for mead sample $6\left(0.484 \mu \mathrm{g} \mathrm{g}^{-1}\right)$ was about 4-fold higher than the highest value found with identical means for regular honey $\left(0.135 \mu \mathrm{g} \mathrm{g}^{-1}\right)$. This is all the more surprising if we assume that candy and mead have undergone some technological treatments (dilution, heating and/or fermentation) and still show PA-values that are well above the average of regular retail honey (Kempf et al. 2008). These few examples clearly demonstrate that a PA-contamination of the used raw material (honey) can be carried on to the final product and significant amounts of PA can still be present. In our studies not all analyzed food categories showed PA-positive samples. Since in any cases the internal standard derivative di-TMS-heliotridine was detected during analysis, the results can be trusted. But, on the other hand we cannot exclude that more sensitive methods or larger sample sets could eventually demonstrate PA carry-over in other honeycontaining food classes as well.

\section{PA carry-over from PA-pollen into honey}

The second part was dedicated to study the potential carry-over of PAs from pollen of PAplants into honey. Recent studies have demonstrated significant amounts of PAs in floral pollen of PA-plants (Boppré et al. 2008; Kempf et al. 2010b). Therefore, PA pollen is discussed as the only source or as major contributor to PA-contamination of honey (Edgar et al. 2002; Boppré et al. 2005; Kempf et al. 2010a). To date, it is unknown, whether the PA-content found in honey is an artifact of the preceding sample preparation (including 
acidic extraction conditions and concentration procedures) or whether PAs are extracted "naturally" from accompanying pollen during the months-long contact starting with the collection of nectar and lasting through honey harvesting, trading of the raw material, packaging, storage, retail up to the final consumption.

If the measured PA-values of honey are directly derived from PA pollen during the sample work-up procedure, this would theoretically offer the possibility to liberate honey from PAcontamination by filtration.

To address these questions we added defined amounts of floral PA pollen derived from Senecio vernalis with a known PA-content $\left(1.85 \pm 0.13 \mathrm{mg} \mathrm{g}^{-1}\right.$, calculated as retronecine equivalents; Kempf et al. 2010b) to PA-free honey. Three typical pollen concentrations were added to PA-free honey, $3 \mathrm{mg} \mathrm{kg}^{-1}$ (representing regular amounts of pollen), $30 \mathrm{mg}$ $\mathrm{kg}^{-1}$ (representing high amounts) and $300 \mathrm{mg} \mathrm{kg}^{-1}$ (serving as a model for honey from sides with massive occurrence of PA-plants and honey harvesting by comb pressing which might contain perga and would therefore result in a unusual high PA-pollen load of the end product). Samples of these honeys were analyzed after defined time points (day 1, 6 wks and 12 wks) with and without honey filtration, using the established procedure for honey samples (Kempf et al. 2008). The conditions of the honey filtration were based on conditions that are used in industrial processes (Paine et al. 1934; Crane 1979; Beckmann 2008) and were down-sized to a laboratory scale. The results of these experiments are summarized in Figure 3.

The findings reveal that the content of PA-pollen correlated with the PA-content that was found for the respective honey sample $(\mathrm{EH} 1$ to $\mathrm{EH} 3$; $\mathrm{EH} 1$ samples being close to the LOQ). In addition, a dependency over time was also recognized for each pollen concentration. If one compares the increasing PA-values over time, here represented by the difference found for day 1 (unfiltered) and the two long term storages after filtration, it becomes obvious that a good portion of the PAs is already liberated from the pollen. 
Hence, the PA-content found in honey cannot solely be based on an artifactual extraction from pollen during the honey work-up (see Fig. 3: honey EH 3 (day 1): $0.094 \mu g^{-1} v s$. EH 3 (12 wks): $\left.0.319 \mu \mathrm{g} \mathrm{g}^{-1}\right)$. Instead, if one compares any "honey pair" (filtered vs. unfiltered) for any given time point there is no difference between these two samples. This indicates that the current extraction techniques for PAs from honey will only detect the PA-portion released from the pollen plus PAs originating from the nectar. Usually, all known work-up procedures for honey include at least one filtration or centrifugation step, hence we could conclude, that the "pollen-bound" PAs in honey are not detected with our general methods (see Fig. 3 EH 2 or 3: theoretical PA-content vs. filtered/unfiltered). This means, at least theoretically, that only a time depending portion of the total PA-content is detected in any given honey, which might cause time depending variations of analytical results. Although, it seems, that the PA-extraction into honey is a rather quick process and the pollen derived PA-amount will eventually reach a plateau (see Fig. 3, EH 3: 6 vs. 12 wks). In addition, and especially true for honeys containing high amounts of PA pollen (e.g. Echium-honeys (Boppré et al. 2005; Boppré et al. 2008; Kempf et al. 2008) a good portion of the total PAamount has not be taken into account yet, but will be ingested as "hidden" or "pollenbound"-PAs and would add, of course depending on the yet unknown bioavailability, to an overall PA-intake.

In addition, the results obtained from the model filtrations clearly demonstrate that honey filtration is not an option to reduce the (free) PA-content of honey (see Fig. 3; EH 2 and 3; 6 and 12 wks: filtered vs. unfiltered). Of course filtration would reduce the (unknown) amount of "hidden/pollen-bound"-PAs. Already after 6 wks of incubation a good portion of the pollen-PA was detected in honey. This could be seen as the PA-transition occurs quite rapidly and is most likely driven by diffusion of the PAs from the pollen into the honey and therefore is irreducible by today permitted methods within the terms of the German honey regulation. 


\section{Conclusions}

The presented data clearly reveals that cross contamination of food through PAcontaminated honey can generally be expected. This can, with some certainty, extrapolated to other possible PA-sources like milk, vegetables, herbs or salads, if these are used as ingredients for recipes. The degree of secondary contamination, at least as here demonstrated for honey, seems less influenced by degradation of the PAs through food processing than by dilution of the original PA-concentration. Here, in this limited study it was surprising, that despite of the dilution of honey, the average PA-content and PApositive rate was very much alike the reported values for honey itself (Kempf et al. 2008; Kempf et al. 2010a). Therefore, the results are a preliminary step on how to address the question which food samples need to be analyzed to establish a solid risk assessment for PAs in the food chain. While many different types of food could be possibly contaminated with PAs and might be worth to be analyzed (like milk, flour, eggs or meat: all characterized by high daily consumption also with a high chance of dilution during the processing, at least in higher developed countries) one should not forget to address food that contains ingredients with known elevated PA-levels like honey or vegetable/salads (BfR 2007; Die Zeit 2010).

In addition we could demonstrate in model studies, that PA pollen are a major source for the PA-content found in honey. The PA-content was direct proportional to the absolute amount of PA pollen. The PA-transfer from pollen to honey is rather quick and therefore regular honey filtration will be no alternative to reduce the PA-content of honey significantly. Whether PA-pollen is the only source of PAs in honey cannot be answered with this experimental setup and is of no relevance for the PA-contamination of honey under those given circumstances. However, it can be assumed that nectar is contributing to the PA-content of honey as well (see Reinhard et al. 2009 and literature cited therein). 


\section{Acknowledgements}

This work was supported by the Deutsche Forschungsgemeinschaft (DFG), Bonn, to P.S. project no. SCHR 211/22-1 and SCHR 211/23-1 and to T.B. project no. BE 3200/1-1, BE 3200/3-1 and BE 3200 / 3-2. 


\section{Literature}

Beckmann K. 2008. Neue Ansätze in der Qualitätsicherung von Honig. [Dissertation] [Dresden]: Technische Universität Dresden.

BfR, Bundesinstitut für Risikobewertung. 2007. Salatmischung mit Pyrrolizidinalkaloidhaltigem Greiskraut verunreinigt. Stellungnahme Nr.028/2007 des BfR vom 10. Januar 2007. Available from: http://www.bfr.bund.de/cm/208/salatmischung_mit_pyrrolizidinalkaloid_haltigem_geiskraut_verunreinigt.pdf (last accessed 23 April 2010).

Boppré M, Colegate SM, Edgar JA. 2005. Pyrrolizidine alkaloids of Echium vulgare honey found in pure pollen. J Agr Food Chem. 53: 594-600.

Boppré M, Colegate SM, Steven M, Edgar JA, Fischer OW. 2008. Hepatotoxic pyrrolizidine alkaloids in pollen and drying-related implications for commercial processing of bee pollen. J Agr Food Chem. 56: 5662-5672.

Crane E. 1979. Honey: A comprehensive survey. London: William Heinemann.

Die Zeit, Newsticker der DPA. 2009. Plus räumt vergifteten Rucola-Salat aus dem Regal. Available from: http://www.zeit.de/newsticker/2009/8/12/HAUPTSTORY-RUCOLAMl22069532xml (last accessed 21 April 2010).

Edgar JA, Roeder E, Molyneux RJ. 2002. Honey from plants containing pyrrolizidine alkaloids: a potential threat to health. J Agr Food Chem. 50: 2719-2730.

European Food Safety Authority (EFSA). 2007. Opinion of the Scientific Panel on Contaminants in the Food Chain on a request from the European Commission related to pyrrolizidine alkaloids as undesirable substances in animal feed. EFSA J447:1-51.

GMBI 2003. Leitsätze für weinähnliche und schaumweinähnliche Getränke in der Fassung vom 27.11.2002. Bekanntmachung vom 23.01.2003. Gemeinsames Ministerialblatt (GMBI). 8-10: 150.

Kempf M, Beuerle T, Bühringer M, Denner M, Trost D, Von der Ohe K, Bhavanam VBR, Schreier P. 2008. Pyrrolizidine alkaloids in honey: Risk analysis by gas chromatographymass spectrometry. Mol Nutr Food Res. 52: 1193-1200.

Kempf M, Reinhard A, Beuerle T. 2010a. Pyrrolizidine alkaloids (PAs) in honey and pollenlegal regulation of PA levels in food and animal feed required. Mol Nutr Food Res. 54: 158-168.

Kempf M, Heil S, Haßlauer I, Schmidt L, Von der Ohe K, Reinhard A, Schreier P, Beuerle T. 2010b. Pyrrolizidine alkaloids in pollen and pollen products. Mol Nutr Food Res. 54: 292-300.

Lüllmann C. 2010. Analysis of Pyrrolizidine Alkaloids (PA) in Honey and Bee Pollen Technical Briefing \& Market Demand. Paper presented at: JRC Workshop on pyrrolizidine alkaloids in food and feed 22-23.02.2010; Bruessels, Belgium. (http://www.feedsafety.org/module/events/file/WorkshopIRMMFeb2010Agenda.pdf)

Paine HS, Gertler SI, Lothrop RE. 1934. Colloidal constituents of honey influence on properties and commercial value. Ind Eng Chem Res. 26: 73-81.

Reinhard A, Jahnke M, Von der Ohe W, Kempf M, Theuring C, Hartmann T, Schreier P, Beuerle T. 2009. Feeding deterrence and detrimental effects mediated by toxic and nontoxic pyrrolizidine alkaloids fed to honey bees. J Chem Ecol. 35: 1086-1095. 


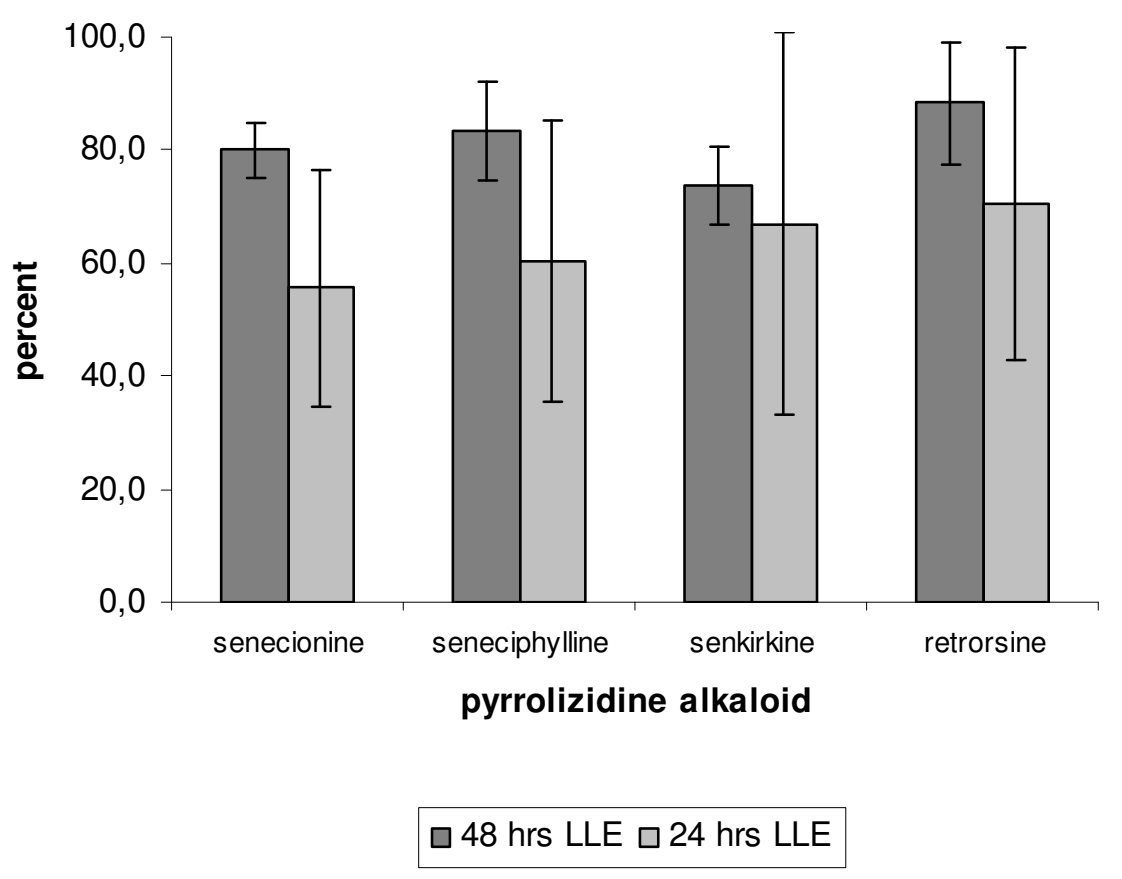

Figure 1: Recovery rates of some individual PAs after $24 \mathrm{hrs}$ and $48 \mathrm{hrs}$ LLE (dichloromethane : pentane 1:2; v/v) with honey matrix. SDs $(n=10)$ are given. 
Figure_2: PA-content of the PA-positive honey-containing foods (calculated as retronecine equivalents); only the foods containing PÂs are listed. SDs $(\bar{n}=\overline{3})$ are given. Samples 1,6 and $9=$ mead; 20 and $24=$ candy; 33,34 and $38=$ fennel honey. Sample numbering is based on the order of analysis. 


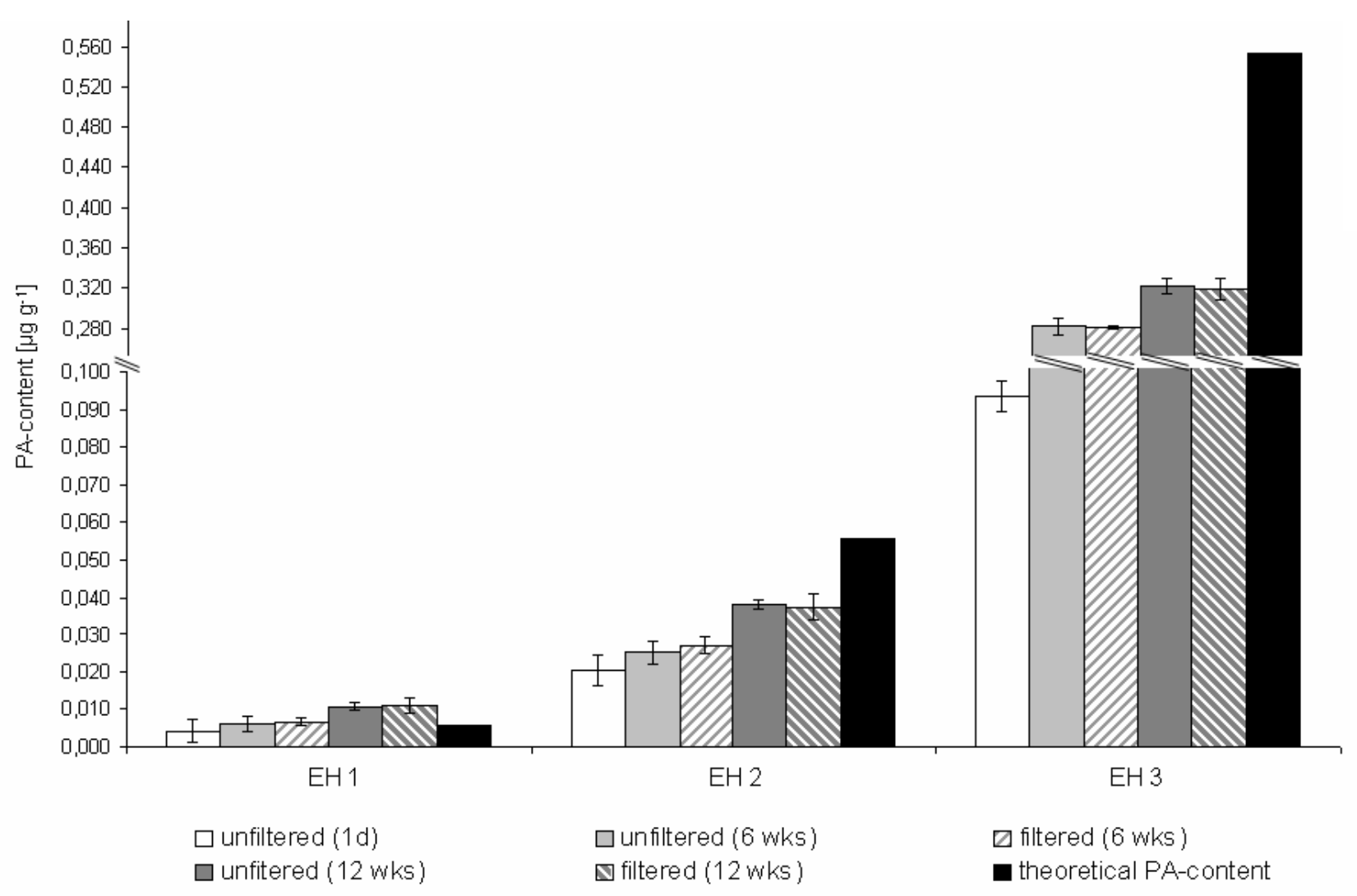

Figure 3: PA-content of the filtered and unfiltered honey samples at defined time points (calculated as retronecine equivalents). SDs $(n=3)$ are given. 